\title{
L'usage du web par l'administration publique : un enjeu de positionnement sur les pratiques des internautes
}

The use of the Internet in public administration: the issue of positioning on the

Internet users practices

Paul Deprez et Yann Bertacchini

\section{CpenEdition}

Journals

Édition électronique

URL : http://journals.openedition.org/communicationorganisation/4969

DOI : 10.4000/communicationorganisation.4969

ISSN : $1775-3546$

Éditeur

Presses universitaires de Bordeaux

Édition imprimée

Date de publication : 1 juin 2015

Pagination : 217-234

ISSN : 1168-5549

\section{Référence électronique}

Paul Deprez et Yann Bertacchini, «L'usage du web par l'administration publique : un enjeu de

positionnement sur les pratiques des internautes », Communication et organisation [En ligne], 47 | 2015, mis en ligne le 01 juin 2018, consulté le 30 avril 2019. URL : http://journals.openedition.org/ communicationorganisation/4969; DOI : 10.4000/communicationorganisation.4969 


\title{
L'usage du web par l'administration publique : un enjeu de positionnement sur les pratiques des internautes
}

\author{
Paul Deprez' et Yonn Bertacchini²
}

\section{Introduction}

Depuis 2005 en France, presque toutes les administrations publiques sont désormais présentes sur le Web et offrent trois types de services administratifs distincts : de l'information, des téléchargements de formulaire et la fourniture complète de services administratifs (Bacache, Bounie, François, 2011). Notre analyse repose sur les nombreux travaux de recherche autour de l'espace public médiatique (Calhoun et al.1992 ; Pailliart et al.1995 ; Boyd 2009; Miège 2010; etc.) et l'expérimentation d'un nouveau territoire, le cyberspace, fondement de l'Espace du savoir, structuré par la mise en connexion de terminaux et de mémoires informatiques, de réseaux de transmission numériques et au sein duquel « tout élément d'information se trouve en contact virtuel avec n'importe quel autre et avec tout un chacun » (Lévy 1995). Il s'agira donc de penser l'expérimentation du cyberspace au profit d'une modernisation de l'action publique non plus dans un paradigme modelé sur des applications émanant de la calculabilité (statistique) mais dans un ordre discursif caractéristique de l'évolution du web 2.0 (Doueihi 2011).

Dans cette perspective, nos travaux s'appuient sur une conceptualisation de cet espace au travers de quatre sphères d'analyse : le web social (Salaun 2009, Sajus 2009 ; Faure, Glassey, Leresche 2010 ; Cordina \& Fayon 2013 ; etc.), le web documentaire (Broudoux 2011, Bachimont et al. 2011, etc.), le web sémantique (Charlet, Laublet, Reynaud 2003 ; Collet 2011; Amar \& Menon 2011, etc.) et le web de l'information (Asdourian 2010 ; Vanbremeersch 2009). L'enjeu de nos travaux consiste non seulement à recenser l'existence ou non de diverses fonctionnalités caractéristiques des espaces du web et en faveur d'une modernisation de l'action publique, dont nous considérons

1 Paul Deprez est docteur en Sciences de l'Information et de la Communication, I3M lab EA 3820, Université de Toulon, paul.deprez@hotmail.fr

2 Yann Bertacchini, MCF-HDR, I3M lab EA 3820, Université de Toulon ; bertacchini@gmail.com 
l'usage des téléprocédures comme stabilisé dans les pratiques routinières de l'administration électronique, mais également interroger les modèles gestionnaires propres au "New Public Management " (Bartoli 2009 ; Pesqueux 2007 ; Osborne et Gaebler 1993 ; Dunleavy 1991) qui supposerait plus de flexibilité dans la gestion des affaires publiques et permettrait aux administrations publiques d'être parcourues par des réseaux d'innovations.

Ainsi, dans une première partie, nous proposons une conceptualisation du cyberspace au travers de différentes sphères du web dit " social », « de l'information ", " sémantique » et « documentaire ». Puis, le second volet de cet article s'intéresse à la mise en parallèle de cette approche du développement de la communication numérique des administrations publiques, par un alignement sur les modes d'engagement des internautes sur le web dont les stratégies de recherche d'information s'échelonnent selon différents comportements.

\section{Typologie des espaces du web : vers une appropriation du contenu fonctionnel}

\section{Le web documentaire}

Le web documentaire correspond à une approche de communication et de publication semblable à celle qui se pratiquait hors ligne : on se contente de mettre à disposition. L'information est descendante et l'interaction n'est pas présente. Ici, le web sert uniquement de lieu d'archivage de contenus numérisés en suivant une logique médiatique où le site Internet est seulement utilisé comme moyen de diffusion. Il existe une réelle publication mais les contenus ne bénéficient d'aucune publicité. En effet, le site ne voit pointer vers lui presque aucun lien et n'existe donc pas dans le territoire du web. Sans liaison, sans visibilité, son impopularité le condamne à l'oubli. Le web documentaire est le règne de l'expert, qui délivre son savoir, sans se soucier de sa préhension par autrui (Vanbremeersch 2009). Les contenus du web documentaire dérivent de l'espace public traditionnel pensant trouver une nouvelle publicité. Pourtant, une fois publié sur le web ce contenu ne circule pas et ne bénéficie donc pas des potentialités offertes par Internet en termes d'échanges et d'interaction. Or, ce qui donne vie à ces contenus ce sont les autres espaces du web : le web de l'information, le web social, le web sémantique. Pour Bruno Bachimont (2011), « le web 1.0 est le web dit documentaire, où les ressources sont publiées sans autre traitement que leur mise en forme et sans autre interactivité que l'activation des liens hypertextuels » (Bachimont et al. 2011).

Cependant, dans le "déluge informationnel ", le web documentaire apparaît comme le symbole d'un renouveau de la production créative où informer, c'est construire une forme, qui prend en compte les caractéristiques communicationnelles du web tout en poursuivant des objectifs d'intérêt général (Broudoux 2011). En ce sens, la captation de l'attention passe par des 
artifices interactifs maintenant l'internaute en immersion dans des univers contraints et addictifs. Ainsi, tout comme les technologies médiatiques, ce web bénéficie des pratiques de montage, ou plutôt de computation, de calcul, de traitement de l'information, qui médiatisent le message somatique ${ }^{3}$ original, au sein d'un processus de création dans lequel la scénarisation occupe une place importante. D'ailleurs, les techniques numériques hantent le média depuis toujours car elles sont « l'absolu du montage, le montage portant sur les plus infimes fragments du message, une disponibilité indéfinie et sans cesse réouverte à la combinaison, au mixage, au réordonnancement des signes » (Levy 1995). Par exemple, l'hypertexte numérique automatise, matérialise les démontages et remontages du sens auxquels se livre le lecteur et en amplifie considérablement la portée.

Les bases de données, systèmes experts, tableurs, hyperdocuments, simulations interactives et autres mondes virtuels sont des potentiels de textes, d'images, de sons ou même de qualités tactiles que des situations particulières actualisent de mille manières. Le numérique retrouve ainsi la sensibilité au contexte des technologies somatiques, tout en conservant la puissance d'enregistrement et de diffusion des médias (Lévy, 1995, p. 58).

Malgré la panoplie d'artifices interactifs dont dispose les éditeurs de contenu, les professionnels sont désormais confrontés à une concurrence vive. En effet, collecte ou émission de l'information originale, traitement, sélection, ne sont plus le monopole des médias dominants, mais sont partagés avec les internautes. Cela appelle des innovations de la part des éditeurs de contenu.

Nous pourrions dire que le web documentaire est celui d'une recherche d'information planifiée dans une approche utilitariste du web. La recherche de contenus est active, volontaire, ciblée et tournée vers une optimisation des ressources. Ici, nous pourrions approcher cet espace de l'activité de curation qui consiste à " repérer divers contenus numériques en fonction d'une thématique donnée, de sélectionner et filtrer les plus pertinents " (Mesguich et al. 2012). Cependant, l'activité du curator consiste également à organiser et structurer ces contenus à travers un dispositif de scénographie et d'en favoriser la diffusion. Or, ce partage n'est possible qu'au travers du web dit « social ». Dès lors, la relation que les publics entretiennent avec les contenus médiatiques de cet espace du web peut s'inscrire dans une logique de recherche d'information où l'utilisateur connaît où trouver le contenu qu'il cherche et n'a pas d'intérêt à le partager. Dans le cas d'une navigation fortuite, plusieurs scénarii peuvent être envisagés quant à la confrontation de l'internaute aux contenus informationnels :

3 Un message somatique est par essence plurimodal car les signes qu'il génère peuvent aussi bien provenir de la parole, de la danse, du chant ou de musique instrumentale. Le dénominateur commun à cette production étant la présence effective, l'engagement, l'énergie et la sensibilité de corps (Lévy, 1995). 
- soit la confrontation d'intérêts communs entre les thématiques des contenus du site web et les goûts de l'internaute;

- soit la désorientation face à un site peu attractif par la faiblesse de scénarisation des contenus et un intérêt trop éloigné de celui de l'internaute ;

- soit la persuasion qui, combinée à un montage des contenus de qualité, pourrait permettre de canaliser l'attention de l'utilisateur ;

Néanmoins, pour certains auteurs, cet espace du web reste l'apanage d'internautes qui, non seulement disposent d'un niveau de certification scolaire élevé et qui, par ailleurs, développent également sur d'autres supports ce type de pratiques du fait notamment d'un intérêt marqué pour l'actualité (Granjon \& Le Foulgoc 2010).

\section{Le web de l'information}

Le Web de l'information partage avec le Web documentaire cette approche non interactive, mais se situe dans l'actualité et l'immédiateté, que l'internaute puisse être informé en temps réel des nouveaux contenus mis en ligne, des informations nouvelles. D'ailleurs, cette immédiateté, qui dépend de l'activité des récepteurs et de la visibilité et accessibilité des contenus, constitue une des principales forces de l'information sur Internet. En effet, les sources de l'information sont désormais multiples et les journalistes, éditeurs professionnels de contenus doivent aujourd'hui composer aux côtés d'internautes actifs qui s'engagent sur le web autrement que dans une posture de spectateurs. Ces derniers effectuent leur propre recoupements, votent, commentent, relient, passent aux amis, exercent une correction, un commentaire, un complément (Vanbremeersch 2009).

Avec le développement d'internet, les « récepteurs » se font producteurs de contenus. En moins de dix ans, archiver, écrire, annoter, commenter, remixer, publier, partager des photos et des vidéos, s'abonner à des flux RSS et échanger des contenus de pair à pair sont devenus des activités relativement communes et pour de nombreux publics. (Monnoyer-Smith 2011 : 164)

Dès lors, l'éditeur d'information professionnelle perd le monopole de l'information au profit de l'internaute lui-même avec lequel il doit désormais composer. Il s'agit donc autant de s'adapter à cette nouvelle culture numérique ou Remix culture, en offrant la possibilité de se positionner sur des pratiques émergentes de personnalisation des interfaces d'accès à l'information, à cette démarche proactive de recherche de l'information, que de miser sur une nouvelle stratégie pour survivre à cette hyperconcurrence. Ainsi, l'utilisation d'outils comme les newsletters, l'alerte par courriel, les flux $\mathrm{RSS}^{4}$ mais aussi les réseaux sociaux représentent autant d'alternatives et de possibilités à

4 Really Simple Syndication. 
offrir à l'internaute pour que ce dernier puisse recevoir les nouveautés d'un site Internet. L'administration électronique dispose donc de fonctionnalités représentatives de l'immédiateté, de l'actualité, pour que l'internaute puisse être tenu informé en temps réel des nouveaux contenus mis en ligne, des informations nouvelles. Nous précisons que le suivi du compte tweeter d'un site ou sa page Facebook représente également une alternative pour recevoir les nouveautés d'un site Internet, à condition d'un renouvellement fréquent des contenus. Cependant, en tant que réseaux sociaux, nous avons préféré étudier ces fonctionnalités dans le cadre du web dit « social » dont les caractéristiques d'échange, partage de contenu et relations entre pairs nous semblent plus représentatives de cet espace numérique.

Sans oublier les réseaux sociaux et la pratique du microblogage, qu'il s'agisse d'un abonnement à la newsletter, à une alerte par courriel ou aux flux RSS. Ces fonctionnalités représentent les principaux outils de collecte et traitement de l'information pour l'internaute en veille. Ces outils présentent également la possibilité de cibler un sujet ou un thème de recherche et participent à une approche utilitariste et planifiée de la recherche d'informations dans laquelle l'utilisateur veut accéder à des ressources précises, dont il connaît ou suppose l'existence. Or, la navigation sur le web ne correspond pas toujours à ce paradigme de la recherche d'informations (Crepel 2008) et il conviendra d'adapter le site aux autres parcours de navigation des internautes.

\section{Le web sémantique}

Le web sémantique constitue une autre voie stratégique de personnalisation des interfaces d'accès à l'information et de navigation des internautes. Il doit permettre de faciliter la recherche d'informations par les humaines. Comme l'explique Laurent Collet :

Un des objectifs du web sémantique est de permettre l'interrogation des moteurs de recherche en langage naturel sans se soucier 1) d'avoir à formuler des requêtes sous forme de mots-clés, 2) à devoir consulter et sélectionner les informations recueillies, voire à les comparer pour obtenir une réponse. Cette sémantisation demande d'ajouter des métadonnées aux contenus affichés et visibles par les internautes sur les pages web. Ces données sont destinées aux algorithmes des robots d'indexation des moteurs de recherche (Collet $2011: 83$ ).

Sans approfondir ou présenter les technologies du web sémantique (langages $R D F^{5}, O W L^{6}$, etc.), l'objectif consiste à développer un web dont le contenu s'adresse, au moins pour partie, aux ordinateurs afin qu'ils puissent aider les utilisateurs humains à se représenter des connaissances et interpréter des contenus (Charlet, Laublet, Reynaud 2003 in Bachimont et al. 2011). Il faut 
donc non seulement être capable d'expliciter le sens d'un contenu mais aussi rendre exploitable cette explication par un traitement informatique. « Le web sémantique naît de la nécessité d'adjoindre des connaissances supplémentaires aux contenus pour améliorer leur traitement » (ibid). D'ailleurs, plutôt que l'appellation web sémantique, d'autres préfèrent parler de données liées ou linked data (Amar \& Menon 2011).

L'indexation des données sur le web a pour but d'interpréter des contenus, de représenter des connaissances et donc permettre un traitement informatique prenant pour objet non seulement le document, en s'inspirant d'informations particulières précisant son type, sa structure, ses termes ou concepts, etc., mais également les stratégies de navigation des utilisateurs et le tagging. Les termes "indexation collaborative " ou encore "indexation sociale " sont également utilisés pour nommer ce processus d'attribution des tags (ou descripteurs) à une ressource pour lui associer une connaissance particulière ou un point de vue original et créer de multiples chemins d'accès à cette ressource, notamment au moyen d'une navigation sur les nuages d'étiquettes, dit aussi "Tags Clouds ", classés par ordre quantitatif (Lambiotte et al. 2006 ; Broudoux 2008 ; Durieux 2010 ; Seilles et al. 2010). À défaut d'avoir vocation à être une plateforme collaborative où les lecteurs indexeraient euxmêmes les contenus à la manière de folksonomy ${ }^{7}$, les sites institutionnels de l'administration publique ont intérêt à utiliser le tagging. En effet, dans le feuilletage des ressources, un certain nombre d'expérimentations a démontré la pertinence d'utilisation des nuages d'étiquettes pour une recherche d'information générale, bien que cette pratique trouve ses limites lorsque la recherche se veut plus précise (Broudoux 2013). Nous tenons à préciser qu'un site Internet constitue un Tags Clouds en tant que résumé sémantique d'une bibliothèque de contenus.

Cette pratique se distingue donc du nuage de mots-clés qui est une représentation visuelle des mots-clés les plus fréquemment utilisés dans un moteur de recherche. D'un côté, le nuage d'étiquettes ou Tags Clouds met en avant le contenu du site et, de l'autre, le nuage de mots-clés reflète les demandes de l'audience au travers d'une représentation visuelle des requêtes effectuées par les internautes selon la répétition d'un mot dans le moteur de recherche Google ou celui propre au site. Comme l'expliquent certains auteurs, " la question est de savoir si les nuages de mots-clés peuvent remplacer les moteurs de recherche en texte intégral et les ontologies de classement ou s'ils leur sont complémentaires. Les recherches effectuées vont davantage dans le sens d'une complémentarité » (Barbe et al. 2010).

7 « Le terme de folksonomy est aujourd'hui couramment utilisé pour décrire la pratique collaborative du tagging et les regroupements d'intérêts que forment les utilisateurs. Alors que l'opération de tagage est individuelle, une dimension collective est introduite par le terme folksonomy " (Broudoux 2013). Tout comme le moteur de recherche ou nuages de mots-clés, les folksonomies représentent une approche possible de l'indexation des ressources sur le web. 
La pratique du tagging alimente une réflexion qui consiste à se demander si les nuages de mots-clés ne semblent pas mieux adaptés aux stratégies de recherche ou découverte d'informations sur le web que des classifications taxinomiques basées sur des ontologies, parfois décrites comme rigides et empreintes d'un certain déterminisme (Crepel 2008). Cependant, il convient de nuancer notre choix. En effet, les nuages de mots-clés, tout comme la pratique des folksonomy, suggèrent un accès aux contenus les plus recherchés ou indexés, ce qui masque les informations les moins visibles. Comme l'expliquent certains chercheurs, ce modèle produit

Un effet d'auto-alimentation des contenus les plus vus par les nuages de mots-clés et l'accentuation d'un phénomène de verticalité restreignant la visibilité à quelques contenus très visités ou très appréciés. Une double dynamique, induite par le système, est à l'œuvre : plus un contenu est lu, plus il est indexé et visible. Et inversement, moins un contenu est visible, moins il est indexé. Nous avons donc un système qui renforce la visibilité des contenus déjà populaires et diminue celle de ceux qui le sont moins (Barbe et al. 2010 : 57).

Pourtant, cette fonctionnalité représente un fort potentiel pour positionner un site face aux stratégies de navigation des internautes et permet de capitaliser des informations sur ces derniers, en comprenant leurs propres logiques de catégorisation et d'association, en identifiant leurs centres d'intérêt et ceux des communautés virtuelles ou réelles auxquelles ils appartiennent (Crepel 2008).

Au travers de l'analyse du web sémantique par l'intermédiaire du moteur de recherche, nuage d'étiquettes et de mots-clés, s'instaurent deux logiques différentes par lesquelles nous pouvons distinguer :

- un courant propre aux sites institutionnels avec une structuration hiérarchique des données et métadonnées pensée et établie par les spécialistes et éditeurs du site (le moteur de recherche, le nuage d'étiquettes) ;

- un courant correspondant à l'espace du web social s'efforçant de placer l'utilisateur au centre de la production des contenus (nuages de mots-clés).

\section{Le web social}

Dès ses débuts le web contenait cette logique sociale de partage de l'information, de discussion ou encore de commentaires directement entre les individus. En revanche, le web social est parfois assimilé au web 2.0 et souhaite caractériser une phase d'évolution, au travers de certaines technologies marquantes (Ruby on Rails, Ajax, etc.), vers plus d'ergonomie, vers une augmentation des équipements des ménages et des pratiques dans l'usage d'Internet, mais également par (Sajus 2009):

- L'émergence de l'individualisme de réseau en rupture avec la dimension collective des forums de discussion par exemple ;

- Des utilisateurs toutes générations confondues ; 
- La standardisation de certains outils du web et l'homogénéité des formats permettent à des milliers de sites de fonctionner en réseau et dialoguer entre eux ;

- La création de communautés virtuelles.

Aujourd'hui, l'utilisateur n'est plus seulement consommateur de données mais désormais au centre de leurs productions et c'est bien cette dimension sociale des applications web 2.0 qui a donné naissance au terme "web social » (ibid.) dit aussi « web participatif » (Faure, Glassey, Leresche 2010). Pour notre part, nous pourrions définir le web social comme un « espace proclamé participatif et interactif, autorisant les interactions au niveau mondial et local, sous forme synchrone ou asynchrone, et caractérisé comme un lieu d'échanges inter et intracommunautaire ». Dès lors, se multiplient les outils de discussion en ligne : messagerie instantanée, mail, forums et newsgroup, documents wiki (même si les discussions justifiant la modification des documents ne se font pas dans le wiki), blogs et outils de réseaux sociaux type Facebook ou Twitter. Dans un contexte où les citoyens et la municipalité n'entretiennent pas le même rapport aux TIC, ce qui est préjudiciable à l'établissement d'un dialogue démocratique (Herbaux \& Bertacchini 2005), le web social constitue un levier nécessaire à la démocratie dialogique, privilégiant à des degrés divers la voie de la discussion et favorisant l'expression du peuple.

Avec un taux de pénétration respectivement de $99 \%$ chez les 15-19 ans, 91,2 \% chez les 20-24 ans, et 75,4 \% chez les 25-29 ans ${ }^{8}$, Facebook s'impose comme l'outil de pratique quotidienne d'échange d'informations pour l'un des publics clef à mobiliser. Il s'agit des jeunes internautes dont la préférence s'oriente vers la communication via un réseau personnel ou interpersonnel (chat, messagerie instantanée, etc.) et non l'utilisation de sites web traditionnels dont la plupart manquent de notoriété numérique (Déprez 2014). On ne peut aujourd'hui passer à côté de ces médias qui ont pris une telle place sur Internet. Pour rappel Facebook, c'est à ce jour plus de 26 millions de visites uniques / mois en France (Médiamétrie 2015) ${ }^{9}$. La pratique du microblogage par Twitter compte elle $30 \%$ des inscrits sur les réseaux sociaux (Médiamétrie 2014) ${ }^{10}$. Aujourd'hui, $70 \%$ des inscrits sur les réseaux sociaux les consultent régulièrement. Par ailleurs, si Facebook et Twitter représentent des réseaux sociaux de premier ordre, nous n'omettons pas de souligner la percée d'Instagram ou encore Google + qui rassemblent respectivement $13 \%$ et $33 \%$ des inscrits à un réseau social (ibid). Il est donc important voire primordial d'être présent sur les réseaux sociaux adéquats à son activité.

\footnotetext{
8 Piedalue Richard, démographie et statistiques Facebook en France, MTLtechno, 10/02/2011 ; http:// mtltechno.com/2011/02/statistiques-demographie-facebook-en-france

$9 \mathrm{http} / /$ www.mediametrie.fr/internet/communiques/1-audience-de-1-internet-en-france-en-novembre-2014. php?id=1189

$10 \mathrm{http} / / /$ www.mediametrie.fr/comportements/communiques/etude-reseaux-sociaux.php?id=1156
} 
Dès lors, cette présence devient un choix politique et une volonté de porter l'information à l'ensemble de la population. Certes, les réseaux sociaux sont devenus des outils de plus en plus incontournables de notre vie de tous les jours mais leurs usages peuvent aussi être d'ordre professionnel et servir aux organisations publiques et privées. Il peut leur être très intéressant d'agréger autour d'elles des groupes d'internautes envers lesquels elles pourront communiquer des informations, permettre à cette communauté de personne de se rencontrer, faire connaissance, échanger et se servir de cette communauté pour écouter les citoyens, recueillir leurs commentaires, lancer des discussions dans un espace de débat, etc. Cependant, nous faisons remarquer que si l'animation d'un réseau social peut parfois être considérée comme une prise de risque en rendant publique l'expression citoyenne et en donnant la parole aux administrés, ces risques s'accompagnent de réelles opportunités en termes de légitimité, d'image, de réactivité face aux avis émis, etc.

Enfin, favoriser la conversation et les échanges, c'est aussi multiplier les outils pour les engager (Poinsot et al. 2010). Dès lors, l'éventail des possibilités doit pouvoir être utilisé : blogs, forum, wikis, sondages, questions, espace de dialogue, etc. Ici, nous souhaitons rappeler les différences entre médias sociaux et réseaux sociaux dans l'espace du web social. En effet, les réseaux sociaux sont au cœur des médias sociaux mais n'en constituent qu'une partie. Ainsi, les médias sociaux peuvent être définis comme « l'ensemble des outils collaboratifs du web 2.0, à savoir les réseaux sociaux, les blogs, les wikis, les forums de discussions. S'y ajoute également une kyrielle d'autres outils de partage (par exemple Skype et outils de chat, univers virtuels). » (Cordina \& Fayon 2013). Ne pas diffuser officiellement son offre d'information sur les médias sociaux dominants (par exemple auprès des jeunes publics) aurait non seulement des conséquences en termes de visibilité, d'attractivité et de représentativité mais irait également à l'encontre des intérêts démocratiques. Ainsi, un éditeur d'information se doit d'actualiser très régulièrement sa politique de présence sur les nouveaux médias.

Cependant, les médias sociaux ne sont pas toujours utilisés par les jeunes générations à des fins d'accès au savoir mais plutôt par mimétisme et pour des communications de type phatique où le changement de statut, les signaux de variation d'humeur, de goûts, d'activités, constitueraient en réalité des signes relationnels (Cardon 2009) pour satisfaire non pas un besoin d'information mais plutôt un besoin d'affirmation dont l'expression est facilitée par le multimédia (Le Deuff 2010 : 42). Dès lors, certains utilisateurs émettent des réticences à voir leur sphère récréative envahie par la présence active de certaines institutions sur les réseaux personnels (Garry 2011 : 6). De plus, pour l'administration électronique, compte tenu de la profusion des médias sociaux lancés ces dernières années sur la toile, il peut lui être difficile d'être présente partout car cette présence nécessite une gestion professionnelle et donc coûteuse. 
Néanmoins, nous souhaitons mettre en exergueles perspectives prometteuses que représentent l'adhésion des internautes à la communauté virtuelle mise en œuvre par une administration publique, par exemple une collectivité territoriale, au travers d'un intérêt commun : la volonté de promouvoir son territoire et revendiquer son identité. Ainsi, une étude (Barabel et al. 2010) montre que l'intégralité des territoires français ont des groupes Facebook et que parmi eux coexistent aussi bien des groupes institutionnels, créés par les collectivités elles-mêmes, avec des groupes personnels, créés par des internautes souhaitant mettre en avant un sentiment de fierté territoriale. Ici, nous faisons remarquer que l'actuel développement de nombreux réseaux virtuels et les perspectives évoquées rendent indispensable l'évolution du référentiel métier des administrations publiques, notamment au travers du community management. L'émergence de ces métiers a plusieurs conséquences. Non seulement, en ce qui concerne le transfert de budgets, puisque « ceux alloués à la communication traditionnelle ont tendance à diminuer, alors que ceux dédiés à la communication numérique progressent ", mais également " la résistance au changement observée parfois chez des salariés et même certains dirigeants »(Cordina \& Fayon 2013). Aussi pour que le community management, et donc l'usage des médias sociaux par l'administration publique, soit couronné de succès, il convient d'impliquer les directions de la communication, la direction générale et selon les spécificités de l'organisation et la stratégie adoptée, d'autres directions ou missions.

\section{L'engagement sur le web : une représentation des pratiques}

Inspiré de l'entreprise, les modèles gestionnaires propres au New Public Management (Bartoli 2009) ; (Osborne et Gaebler 1992) supposent en réalité plus de flexibilité dans la gestion des affaires publiques et permettent aux administrations publiques d'être parcourues par des réseaux d'innovations. A l'heure d'une démocratisation d'Internet à la majeure partie de la population (Lemoine 2012), il s'agissait donc de proposer un certain nombre de fonctionnalités offertes aux administrations publiques pour ne pas les voir s'éloigner des internautes dont les comportements sur le web s'échelonnent à différents degrés (Li \& Bernoff 2008 ; Anderson, Bernoff, Reitsma, Sorensen 2010 ; Cabinet Altimeter 2010 dans Mesguich et al. 2012) :

- Les collecteurs : face au phénomène d'infobésité, certains utilisateurs du web mobilisent des outils comme les flux RSS, la newsletter ou le moteur de recherche afin de repérer et sélectionner des informations parmi la surabondance de contenus. Sur les plateformes collaboratives en ligne, ils sont à l'origine de folksonomy puisque ce sont eux qui assignent des étiquettes ou tags aux contenus qu'ils diffusent ; 
- Les créateurs et sharers : ce sont les utilisateurs qui publient et modifient les wikis ${ }^{11}$, créent leurs propres blogs, forum en ligne ou site web, rédigent des articles, partagent vidéos, photos et musiques avec leur communauté virtuelle et participent donc à la viralité des contenus sur le web. Le livre blanc de la firme Aimia12 évoque le terme de "cliquers " pour qualifier l'engagement des internautes sur le web principalement actifs sur Facebook, seul réseau sur lequel ils sont présents, avec le partage de statuts, photos et les commentaires. En 2011, selon l'institut américain GfK Mediamark Research E Intelligence ${ }^{13}$, ils représenteraient $6 \%$ des usagers du web ;

- Les critiqueurs ou causeurs : ils représentent les utilisateurs qui expriment leur opinion en laissant des commentaires sur les blogs, les forums en ligne. Si Charlene Li et Josh Bernoff (2008) intègrent à cette catégorie les créateurs de wikis, nous préférons plutôt les considérer comme partie intégrante du degré le plus élevé sur la " pyramide de l'engagement " (cf. figure $\mathrm{n}^{\circ} 1$ ).

Bien que nous distinguions la catégorie des " collecteurs " de celle des " créateurs " et "sharers ", en termes d'usages, nous pourrions qualifier l'ensemble de ces utilisateurs du web de «curator». D'origine anglo-saxonne, le terme désigne le responsable des collections d'un musée dont le travail consiste à "veiller » sur les œuvres. Par exemple, en les sélectionnant pour les mettre en valeur lors d'une exposition. Cependant, ce terme a été repris pour décrire cette pratique du web qui consiste à identifier les sources, trier et collecter les informations, agencer les contenus puis les diffuser sur les réseaux ou communautés auxquels le curator appartient (Mesguich et al. 2012). Selon le cabinet Altimeter ${ }^{14}$ (2010), les curators, en tant que créateurs de Wikis, modérateurs de forums ou animateur de communauté virtuelle, rédacteurs d'articles, représenteraient à peine $1 \%$ des utilisateurs du web. En y ajoutant la catégorie des " collecteurs ", le cabinet Forrester ${ }^{15}$ (2008, 2010) évalue à $20 \%$ des internautes, les utilisateurs du web qui pratiquent la curation. Cependant, par extension, chaque utilisateur d'un réseau social, d'un blog ou d'une plateforme collaborative (exemple : Flickr), est un curator en devenir, à partir du moment où il choisit de diffuser des contenus thématiques (Mesguich et al. 2012).

Comme l'explique Camille Alloing (2012), il convient de ne pas confondre l'activité du curator de celle du veilleur. Tout d'abord, selon lui, ce qui motiverait un curator, c'est l'accomplissement de soi et non pas la réponse

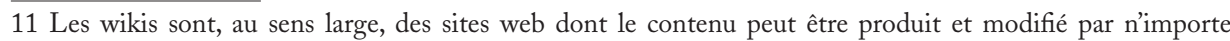
quel utilisateur, de manière collaborative et collective. Le plus célèbre et probablement le plus utilisé étant la Wikipédia (Roth et al. 2008).

12 http://www.pamorama.net/wp-content/uploads/2012/06/Aimia-Social-Media-White-Paper-6-types-of-social-mediausers.pdf

$13 \mathrm{Ibid}$

$14 \mathrm{http}: / /$ www.altimetergroup.com/2010/01/socialgraphics-webinar-slides-and-recording-now-available.html 15 Voir Li \& Bernoff, 2008 ; Anderson, Jacqueline, Bernoff, Josh, Reitsma, Reineke, Sorensen Erica (Cabinet Forrester), (2010) ; A Global Update Of Social Technographics An Empowered Report: Social Media Growth Is Centered On Social Networking. 
aux besoins d'un commanditaire identifié. Pourtant, il convient de nuancer cette affirmation puisque dans le cas de l'analyse des wikis, la "motivation altruiste ", correspondant à " la contribution bénévole à un bien public ", est également soulignée comme l'une des caractéristiques du créateur de contenus (Roth et al. 2008). Ensuite, le curator se différencie du veilleur car il ne suit pas une méthodologie précise (objectifs, besoins du commanditaire) qui conditionnerait le choix de l'outil. Enfin, la veille passe par l'analyse des informations collectées alors que la curation peut parfois s'appuyer uniquement sur une reprise immédiate de prescriptions d'autres curators avec le risque de rediffuser des informations erronées (Alloing 2012). Nous sommes ici face à l'une des caractéristiques des sphères publiques médiatées décrite par Danah Boyd (2009) : la « reproductibilité ».

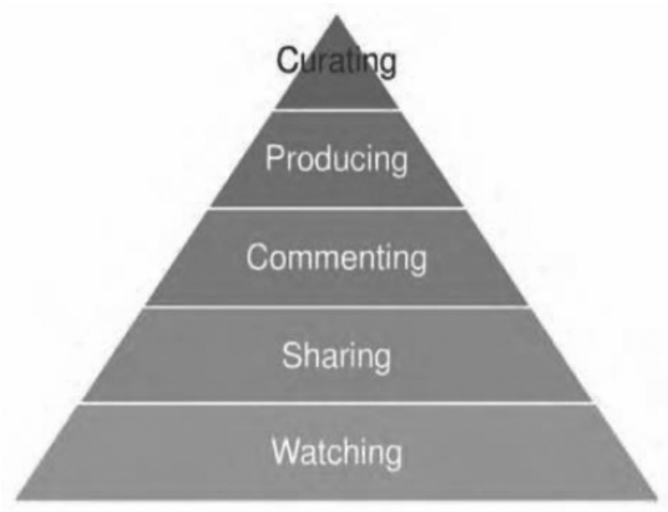

Figure 1 : La pyramide de l'engagement présentée par le cabinet Altimeter ${ }^{16}$ dans le cadre du webinar "Understanding Your Customers' Social Behaviors", janvier 2010

- Les spectateurs, qui se limitent à la consommation de contenus et vont donc être plus attentifs à la scénarisation et au montage. Dans le déluge informationnel, la captation de l'attention passe par des artifices interactifs maintenant l'internaute en immersion dans des univers contraints et addictifs. Ici, la recherche d'information sur le web suppose un type de navigation où la recherche de contenus est soit active, volontaire, ciblée et tournée vers une optimisation des ressources ${ }^{17}$, soit souple et opportuniste selon le principe de " sérendipité » ${ }^{18}$ (Quintarelli 2005 ; Auray 2007). En effet, « les occasions d'un saisissement des contenus d'actualité sont aussi le fait de pratiques fortuites

16 http://www.altimetergroup.com/2010/01/socialgraphics-webinar-slides-and-recording-now-available.html 17 Cette approche reste l'apanage d'internautes qui, à l'évidence, disposent d'un niveau de certification scolaire élevé et qui, par ailleurs, développent également sur d'autres supports ce type de pratiques du fait notamment d'un intérêt marqué pour l'actualité (Granjon et Le Foulgoc, 2010).

18 Navigation sans objectif prédéfini qui va s'effectuer à partir des réseaux sociaux, des réseaux de métadonnées (folksonomy, nuages de mots-clés, Tags Clouds), de la réception d'un courriel comprenant un lien hypertexte, etc., dans laquelle on peut considérer l'internaute comme ouvert à l'offre. 
qui s'appuient sur des prises contextuelles inattendues, opportunes, qui n'ont pas grand-chose de commun avec une démarche proactive de recherche d'information » (Granjon et Le Foulgoc 2010);

- Les membres ou joiners : avec ce comportement des utilisateurs, le web se transforme en gigantesque club où l'appartenance à une communauté devient une pratique de plus en plus généralisée. D'ailleurs, l'étude conduite par Aimia ${ }^{19}$ évoque non seulement la catégorie des « cliquers» mais y adjoint également les « onlookers » qui représenteraient $16 \%$ des usagers du web social et dont l'engagement est caractérisé par une présence sur plusieurs réseaux en même temps, mais qui ont une participation très limitée avec des publications peu fréquentes. Ils sont présents pour suivre les activités de leurs contacts (famille, collègues, amis, etc.). L'étude, menée par le cabinet Forrester Research et intitulée "An Empowered Report: Social Media Growth Is Centered On Social Networking ", démontre une évolution notable de l'activité sur le web où le « join " prime désormais sur le "search » (Anderson, Bernoff, Reitsma, Sorensen 2010) pour la tranche d'âge 12-26 ans (cf. graphique $n^{\circ} 1$ page suivante).

\section{Conclusion}

La réflexion engagée dans le présent article est intimement liée à l'hypothèse d'alignement des acteurs proposée par Flichy et Dagiral (2004) lorsqu'ils postulent que l'un des principaux obstacles à la mise en place de l'e-administration est la difficile mise en cohérence des acteurs. Selon eux, la réussite d'un projet technique nécessite d'aligner les acteurs concernés autour d'un projet commun, notamment par un accompagnement à la matérialisation des échanges. Ici, notre proposition théorique consiste à comprendre les possibilités d'appropriation des TIC par l'administration publique sans entrer dans une sorte de «technologisme » (Mucchielli 1996) ou idéologie de la technique, qui postule que la technique serait la principale cause de tous les changements à divers niveaux ou qu'elle constituerait à elle seule le projet. Il s'agit avant toute chose de définir en interne l'intérêt d'organiser un usage des TIC au service d'une politique publique, et non pas l'inverse. Par ailleurs, l'engagement sur le web nécessite des compétences particulières et donc un budget à part entière. Pour conclure, nous pourrions ouvrir le débat et proposer de compléter l'analyse de façon empirique afin d'étudier l'appropriation et l'usage par les administrations publiques des différentes fonctionnalités discutées au sein de cet article.

19 http://www.pamorama.net/wp-content/uploads/2012/06/Aimia-Social-Media-White-Paper-6-types-ofsocial-media-users.pdf 


\begin{tabular}{|c|c|c|c|c|}
\hline \multicolumn{2}{|l|}{ What people are doing } & \multicolumn{3}{|c|}{ Who participates (U.S. online users) } \\
\hline & & $\begin{array}{c}\text { Young Teens } \\
12 \text { to } 17 \\
1\end{array}$ & $\begin{array}{c}\text { Youth } \\
18 \text { to } 21 \\
\end{array}$ & $\begin{array}{c}\text { Generation Y } \\
22 \text { to } 26 \\
\end{array}$ \\
\hline $\begin{array}{r}\text { Creators publish } \\
\text { Web pages, write blogs, } \\
\text { upload videos to sites } \\
\text { like You Tube. }\end{array}$ & 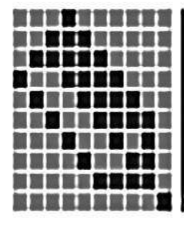 & 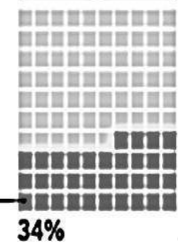 & 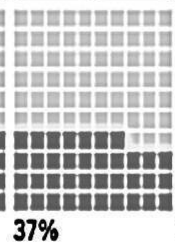 & 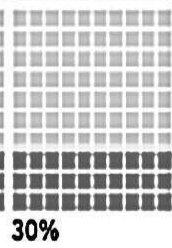 \\
\hline $\begin{array}{r}\text { Critics comment on } \\
\text { blogs and post } \\
\text { ratings and } \\
\text { reviews. }\end{array}$ & 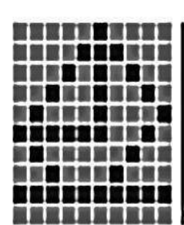 & 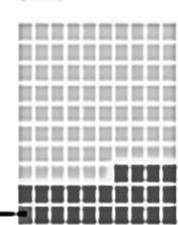 & 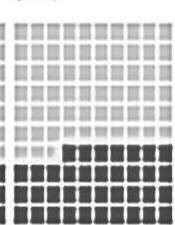 & 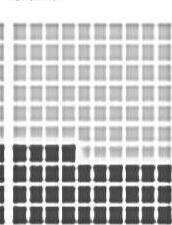 \\
\hline & 列 & $24 \%$ & $37 \%$ & $34 \%$ \\
\hline $\begin{array}{r}\text { Collectors use } \\
\text { Really Simple } \\
\text { Syndication (RSS) } \\
\text { and tag Web pages } \\
\text { to gather information. }\end{array}$ & 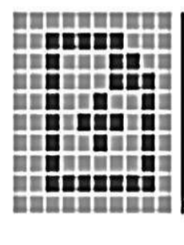 & 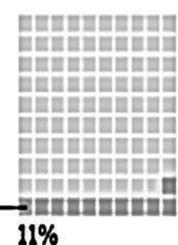 & 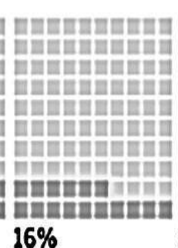 & 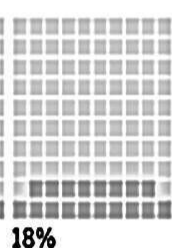 \\
\hline $\begin{array}{l}\text { Joiners use social } \\
\text { networking sites. }\end{array}$ & 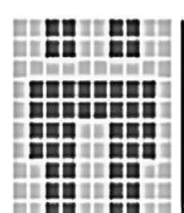 & 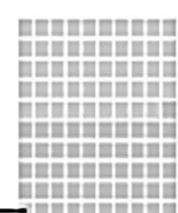 & 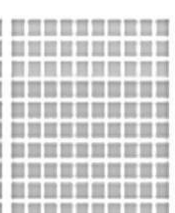 & 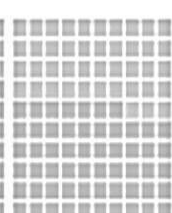 \\
\hline & & $51 \%$ & $70 \%$ & $57 \%$ \\
\hline $\begin{array}{r}\text { Spectators read } \\
\text { blogs, watch peer- } \\
\text { generated videos, and } \\
\text { listen to podcasts. }\end{array}$ & 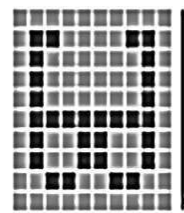 & 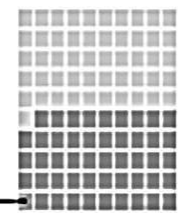 & 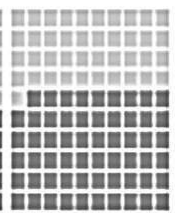 & 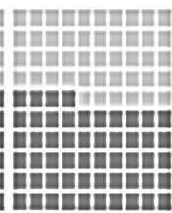 \\
\hline & & & $59 \%$ & $54 \%$ \\
\hline
\end{tabular}

Graphique 1 : Comportement des utilisateurs du web âgés de 12 à 26 ans selon leur degré d'engagement (Anderson, Bernoff, Reitsma, Sorensen, 2010) 


\section{BIBLIOGRAPHIE}

AMAR M., MENON, B. (2011), "Quelle donnée ? ", Documentaliste-Sciences de l'Information, Vol. 48, p. 20-20.

ANDERSON, J., BERNOFF, J., REITSMA, R., SORENSEN, E. (2010), A Global Update Of Social Technographics An Empowered Report: Social Media Growth Is Centered On Social Networking, Forrester.

AURAY, N. (2007), «Folksonomy : the New Way to Serendipity». Communication and strategies, n 65 , p. 67-91.

ASDOURIAN Bruno (2010), Communication relation et médias sociaux : étude du cas de la sensibilisation aux feux de forêt, Thèse soutenue à l'Université d'Aix Marseille.

BACACHE-BEAUVALLET M., BOUNIE D., FRANÇOIS A. (2011), « Existe-til une fracture numérique dans l'usage de l'administration en ligne ? ", Revue économique, 2011/2. Vol. 62.

BACHIMONT B., et al. (2011), "Enjeux et technologies : des données au sens ", Documentaliste-Sciences de l'information, Vol. 48, p. 24-41.

BARABEL M., et al. (2010), «Les médias sociaux au service du marketing territorial : une approche exploratoire », Management E Avenir, n 32, p. 233-253.

BARBE L.et al. (2010), «Un enjeu de société », Documentaliste-Sciences de l'Information, Vol. 47, p. 56-67.

BARTOLI A. (2009), Management dans les organisations publiques. $3^{\mathrm{e}}$ édition, Paris, Dunod, 405 p.

BERTACCHINI Y. (2010), «Intelligence territoriale : une lecture rétro-prospective », Revue internationale d'intelligence économique, 2010/1 Vol. 2, p. 65-97.

BOYD D. (2009), Sorti du contexte : la sociabilité des adolescents américains dans les espaces publics en réseau, thèse "Information Management and Systems » soutenue à l'Université de Berkeley.

BROUDOUX E. (2013), «Quelles lectures du tagging? Modélisation, techniques et usages ", Document numérique, Vol. 16, p. 55-71.

BROUDOUX E. (2011), "Le documentaire élargi au web » in " Le(s) Multimédia(s) », Les Enjeux de l'information et de la communication, déc. 2011.

BROUDOUX, E. (2008), «Indexation collaborative : entre gain informationnel et déperdition conceptuelle ? ", Document numérique et société, 17-18 novembre, Paris, Cnam.

CALHOUN C. (éd.), (1992), Habermas and the public sphere. Cambridge (Mass.), MIT Press, coll. Studies in contemporary German social thought.

CARDON D. (2009), «L'identité comme stratégie relationnelle », Hermès, La Revue, $\mathrm{n}^{\circ} 53$, p. 61-66.

COLLET L. (2011), « La presse écrite sur le web et ses lecteurs comme limites aux techniques de personnalisation de l'information ", Document numérique, Vol. 14, p. 81-102.

CORDINA P., FAYON D. (2013), Community management : fédérer des communautés sur les médias sociaux, Montreuil, Pearson.

CREPEL M. (2008), « Les Folksonomies comme support émergent de la navigation sociale et de structuration de l'information sur le web ", Réseaux, n 52, p. 169-204. 
DÉPREZP. (2014), Collectivités territoriales et Développement durable : contribution des technologies de l'information, et de la communication, à la dimension participative d'une politique publique. Lecture d'un projet cyberdémocratique issu d'une démarche d'Intelligence Territoriale. Thèse soutenue à l'Université de Toulon.

DOUEIHI M. (2011), Pour un humanisme numérique, Paris, Seuil, La Librairie du XXI ${ }^{e}$ siècle, $192 \mathrm{p}$.

DURIEUX V. (2010), «Collaborative tagging et folksonomies ", Lorganisation du web par les internautes. Les Cabiers du numérique, Vol. 6, p. 69-80.

FAURE A. et al. (2010), "Démocratie participative et Démocratie différentielle ", Pôle Sud, nं 32, p. 11-23.

FLICHY, P., DAGIRAL, E. (2004), « L'administration électronique : une difficile mise en cohérence des acteurs ", Revue française d'administration publique, 2004/2 $\mathrm{n}^{\circ} 110$, pp. 245 à 255.

FLICHY P. (2008), «Internet et le débat démocratique », Réseaux, n 150, p. 159-185.

GARRY O. et al. (2011), « Méthodes techniques et outils », Documentaliste-Sciences de l'information, Vol. 48, p. 4-11.

GRANJON F., Le FOULGOC A. (2010), " Les usages sociaux de l'actualité ", L'Expérience médiatique des publics internautes. Réseaux, n 160-161.

HERBAUX P., BERTACCHINI Y. (2005), " Les TIC, leviers de gouvernance territoriale ", International Journal of Information Sciences for Decision Making, $\mathrm{n}^{\circ} 21$.

LAMBIOTTE R., AUSLOOS M. (2006), «Collaborative tagging as a tripartite network », Lecture Notes in Computer Science, vol. 3993, p. 1114-1117.

LE DEUFF O. et al. (2010). Une évolution des comportements. DocumentalisteSciences de l'Information, Vol. 47, p. 42-45.

LEVY P. (1995), Lintelligence collective : Pour une anthropologie du cyberspace, Paris, La Découverte, 243 p.

LEMOINE J-F. (2012), "À la recherche d'une meilleure compréhension du comportement des internautes ", Management \& Avenir, n 58, p. 116-119.

LI C., BERNOFF J. (Cabinet Forrester), (2008), Groundswell: Winning in a world transformed by social technologies, Harvard Business Press, p. 286.

MESGUICH V. et al. (2012), "Enjeux et dimensions », Documentaliste-Sciences de l'information, Vol. 49, p. 24-45.

MONNOYER-SMITH L. (2011), « La participation en ligne, révélateur d'une évolution des pratiques politiques? », Participations, n 1, p. 156-185.

MUCCHIELLI A. (1996), « Pour des recherches en communication », Communication et organisation, 10, p. 2-16.

OSBORNE D., GAEBLER T. (1992), Reinventing Government : How the Entrepreneurial Spirit Is Transforming the Public Sector, Reading, MA : Addison-Wesley.

PAILLIART I. (dir.), (1995), L'espace public et l'emprise de la communication. Grenoble, Ellug, $211 \mathrm{p}$.

PEREA F. (2010), «L'identité numérique : de la cité à l'écran. Quelques aspects de la représentation de soi dans l'espace numérique ", Les Enjeux de l'information et de la communication, Volume 2010, p. 144-159. 
POINSOTT. et al. (2010), «Du collaboratif au social :l'avènement de la conversation », Documentaliste-Sciences de l'information, Vol. 47, p. 26-37.

QUINTARELLI E. (2005), Folksonomies : power to the people. Proceedings of the 1st ISKOI, UniMIB Metting, Italy.

ROTH C. et al. (2008), "Démographie des communautés en ligne, Le cas des wikis », Réseaux, n 152 , p. 205-240.

SAJUS B. (2009), "Est-ce que j'ai une tête de deux point zéro ? ", DocumentalisteSciences de l'information, Vol. 46, p. 30-31.

SEILLES A. et al. (2010), "L'annotation discursive et sémantique pour la pratique de "débats 2.0" ", Document numérique, Vol. 13, p. 153-177.

VANBREMEERSCH N. (2009), De la démocratie numérique, Le Seuil, Paris.

Résumé : À l'heure où l'ensemble des administrations sont présentes sur le web et proposent de l'information ainsi que des téléchargements de formulaires, l'article examine les possibilités offertes à l'administration électronique pour s'adapter aux comportements des internautes dont les stratégies de recherche d'information s'échelonnent selon différents degrés. Il s'agit là autant de proposer une conceptualisation du cyberspace au travers de différentes sphères du web dit « social ", " de l'information ", " sémantique » et « documentaire ", que de questionner les modèles gestionnaires propres au new public management. Il s'agit ainsi de proposer une approche du développement de la communication numérique des administrations publiques, aligné avec les modes d'engagement des internautes sur le web.

Mots-clés : Cyberspace, administration électronique, web social, web sémantique, web de l'information, web documentaire.

Abstract: Because we know the behavior of Internet users, particularly information search strategy, can be ranged on various degrees and all governments are present on the web offering both information and downloads of forms, this article examines possibilities of e-government to adapt to. We both consider, a conceptualization of cyberspace through different areas of uses "Social Web", "Web of Information", "Semantic web" and "Documentary" one and address, to managers own models of New Public Management, some questions. The aim is to propose a development approach of digital communication government in relation with patterns of involvement of web users.

Keywords: Cyberspace, e-government, social web, semantic web, web information, web documentary. 
